\title{
SPECIAL TRAIN ALGEBRAS ARISING IN GENETICS
}

\author{
by H. GONSHOR
}

(Received 18th January 1959)

(Received in revised form 22nd December 1959)

\section{Introduction}

Ever since Mendel promulgated his famous laws, probability theory and statistics have played an important role in the study of heredity (9). Etherington introduced some concepts of modern algebra when he showed how a nonassociative algebra can be made to correspond to a given genetic system $(\mathbf{1}, \mathbf{4})$. The fact that many of these algebras have common properties has led to their study from a purely abstract point of view $(2,3,5,6,11,12)$. Furthermore, the techniques of algebra give new ways of attacking problems in genetics such as that of stability.

We shall prove a stability theorem on special train algebras and apply it to several genetic systems.

\section{A Stability Theorem for Special Train Algebras}

For our purpose, the basis form of the definition will be most useful. A special train algebra is a commutative algebra for which there exists a basis $a_{0}, a_{1}, \ldots, a_{n}$ with a multiplication table of the following kind:

$$
\begin{aligned}
& a_{i} a_{j}=\Sigma x_{i j k} a_{k} \text { where } \\
& \quad x_{000}=1, \\
& \quad \text { for } k<j, x_{0 j k}=0, \quad(2) \\
& \quad \text { for } i, j>0 \text { and } k \leqq \max (i, j), x_{i j k}=0,
\end{aligned}
$$

and furthermore all powers of the ideal $\left\{a_{1}, a_{2}, \ldots, a_{n}\right\}$ are ideals. (The powers $I^{r}$ of an ideal $I$ are defined inductively by $\left.I^{r}=I^{r-1} I\right)$.

In this section we shall require conditions (1), (2) and (3) only. It follows easily that $\left\{a_{1}, a_{2}, \ldots, a_{n}\right\}$ is nilpotent and also that the map which sends each element into its coefficient of $a_{0}$ is the only non-trivial homomorphism of the algebra into the coefficient field. The $x_{0 j j}$ (abbreviated $\lambda_{j}$ ) will be called the train roots of the algebra. They are the characteristic roots of the operator which is multiplication by $a_{0}$. Necessarily $\lambda_{0}=1$. (They include the principal train roots defined in (1), perhaps with repetitions, but it is important to note that they may also include $\frac{1}{2}$ even when this is not a principal train root.)

Theorem 2.1. Every special train algebra which has no train root satisfying $2 \lambda=1$ has a unique non-zero idempotent.

Proof. We shall construct the $x_{i}$ inductively so that $\left(\sum_{i=0}^{m} x_{i} a_{i}\right)^{2}$ has the 
form $\sum_{i=0}^{m} x_{i} a_{i}+\sum_{i=m+1}^{n} y_{i} a_{i}$. Since $x_{000}=1, x_{0}$ must be 1 for a non-zero idempotent. Suppose $x_{0}, x_{1}, \ldots, x_{n}$ have been chosen. Then

$$
\left(\sum_{i=0}^{m} x_{i} a_{i}+z a_{m+1}\right)^{2}=\sum_{i=0}^{n} x_{i} a_{i}+\sum_{i=m+1}^{r} y_{i} a_{i}
$$

for some $y$ 's. This follows since from (2) and (3) the coefficient of $a_{i}$ for $i \leqq m$ in a product is independent of the coefficients of $a_{m+1}$ in the factors. It remains only to equate the coefficients of $a_{m+1}$. Since from (3) $a_{m+1} a_{i}$ for $i \neq 0$ has coefficient of $a_{m+1}$ equal to 0 , the coefficient of $a_{m+1}$ will have the form $2 \lambda_{m+1} z+f\left(x_{1}, x_{2}, \ldots, x_{m}\right)$. Since $2 \lambda_{m+1} \neq 1$, the equation in $z, 2 \lambda_{m+1} z+$ $f\left(x_{1}, x_{2}, \ldots, x_{m}\right)=z$ has a unique solution which is taken as $x_{m+1}$. This proves the existence and uniqueness simultaneously.

Note. For a coefficient field of characteristic 2 the above condition is always satisfied. Otherwise, the condition may be stated in the form, no $\lambda=\frac{1}{2}$.

Similar theorems may be proved in cases where several of the $\lambda$ 's are equal to $\frac{1}{2}$. The case where $\lambda_{1}=\frac{1}{2}$ and all other $\lambda$ 's are distinct from $\frac{1}{2}$ occurs frequently in genetics. By the same technique as before it is easy to show that non-zero idempotents exist only if $x_{001}=0$ and in that case a one parameter family of idempotents exists. (In fact, $x_{1}$ may be chosen at will, and the other $x$ 's are uniquely determined as before.) Similarly if $r$ of the $\lambda$ 's, and no more, are equal to $\frac{1}{2}$, it can happen that there is an $r$-parameter family of idempotents. This occurs in examples connected with multiple alleles and with linkage (3).

Henceforth, we shall consider algebras over the reals. We may then define $\sum_{m=0}^{n} x_{m i} a_{m} \rightarrow \sum_{m=0}^{n} x_{m} a_{m} \Leftrightarrow x_{m i} \rightarrow x_{m}$ for all $x$. It is clear that convergence defined this way is independent of the basis used. In order to state the next theorem it will be convenient to define two concepts. The "weight" of an element is its image in the unique homomorphism of the algebra onto the reals. The " plenary powers" of an element $A$ are defined as $A_{0}=A, A_{n+1}=A_{n}^{2}$.

Theorem 2.2. The sequence of plenary powers of an element of weight 1 in a special train algebra whose train roots other than $\lambda_{0}=1$ all have absolute value less than $\frac{1}{2}$ tends to an idempotent.

Proof. Suppose $A_{i}=\sum_{m=0}^{n} x_{m i} a_{m}$. It is required to show that $x_{m i} \rightarrow x_{m}$ where $x_{m}$ is defined as in Theorem 2.1. It is clear that $x_{0 i}=1$ for all $i$. Assuming $x_{1 i} \rightarrow x_{1}, x_{2 i} \rightarrow x_{2}, \ldots, x_{m i} \rightarrow x_{m}$, we shall show that $x_{m+1, i} \rightarrow x_{m+1}$. As in the proof of Theorem $2.1, x_{m+1, i+1}=2 \lambda_{m+1} x_{m+1, i}+f\left(x_{1 i}, \ldots, x_{m i}\right)$ where $f$ is a function depending only on the multiplication table of the algebra. Since $f$ is a quadratic function $f\left(x_{1}, \ldots, x_{m i}\right) \rightarrow f\left(x_{1}, \ldots, x_{m}\right)$. The result now reduces to the following theorem in analysis. Let $\left(a_{n}\right)$ and $\left(b_{n}\right)$ be two sequences satisfying 
$a_{n+1}=\lambda a_{n}+b_{n}$ for some $\lambda$ with $|\lambda|<1$ and let $b_{n} \rightarrow b$; then $\left(a_{n}\right)$ converges to the limit $a$ determined by $a=\lambda a+b$.

Write $a_{n}=a+u_{n}, b_{n}=b+y_{n}$. We have $u_{n+1}=\lambda u_{n}+y_{n}$, where $|\lambda|<1$ and $y_{n} \rightarrow 0$, and have to prove that $u_{n} \rightarrow 0$. Since

$$
u_{n+1}=\lambda^{n} u_{1}+\lambda^{n-1} y_{1}+\lambda^{n-2} y_{2}+\ldots+y_{n}
$$

if we define $z_{n}=\lambda^{n-1} y_{1}+\lambda^{n-2} y_{2}+\ldots+y_{n}$, it will suffice to show that $z_{n} \rightarrow 0$. In this form the result is contained in Knopp ((10), p. 76, 9(b)).

The theorem may be extended to cases where several of the $\lambda$ 's are equal to $\frac{1}{2}$. If $\lambda_{1}$ only $=\frac{1}{2}$, then the theorem is still valid if $x_{001}=0$; otherwise the sequence $x_{1 i}$ is an arithmetic progression which certainly does not converge.

\section{Mutation Algebras}

If the simplest case is considered with mutation rates $r$ and $s$, the gametic algebra has basis $D$ and $R$ and the multiplication table:

$$
\begin{aligned}
D^{2} & =(1-r) D+r R, \quad R^{2}=s D+(1-s) R, \\
D R & =\frac{1}{2}\{(1-r) D+r R\}+\frac{1}{2}\{s D+(1-s) R\}=\frac{1}{2}(1-r+s) D+\frac{1}{2}(1-s+r) R .
\end{aligned}
$$

It can be verified by direct computation that if $a$ and $b$ are chosen as a basis where $a=D$ and $b=D-R$, the table takes on the simplified form:

$$
a^{2}=a-r b, \quad a b=\frac{1}{2}(1-r-s) b, \quad b^{2}=0 .
$$

Thus the algebra is a special train algebra. (Note that the basis is independent of $r$ and s.) Theorems 2.1 and 2.2 can therefore be applied. By theorem 2.1 there is a unique non-zero idempotent unless $r+s=0$. By theorem 2.2 the plenary powers of an element of weight 1 approach this idempotent for $0<r+s<2$.

Although the algebra is defined for all real $r$ and $s$, the corresponding genetic system has meaning only for $0 \leqq r \leqq 1,0 \leqq s \leqq 1$. Since it is known that in the case $r=s=0$ (no mutations) stability is achieved in the second generation, this proves that the gene distribution approaches a stable distribution unless possibly when $r=s=1$. This case is clearly unstable since $D^{2}=R$ and $R^{2}=D$. More generally, the plenary powers of $\alpha D+\beta R$, where $\alpha+\beta=1$, oscillate between $\alpha D+\beta R$ and $\beta D+\alpha R$, only the distribution $\frac{1}{2} D+\frac{1}{2} R$ being one of unstable equilibrium.

The zygotic algebra has the table

$$
\begin{aligned}
D^{2} & =(1-r)^{2} D+2(1-r) r H+r^{2} R, \quad R^{2}=s^{2} D+2(1-s) s H+(1-s)^{2} R, \\
D H & =\frac{1}{2}(1-r)(1-r+s) D+\frac{1}{2}[1+(1-2 r)(r-s)] H+\frac{1}{2} r(1+r-s) R, \\
R H & =\frac{1}{2} s(1-r+s) D+\frac{1}{2}[1+(1-2 s)(s-r)] H+\frac{1}{2}(1-s)(1+r-s) R, \\
D R & =(1-r) s D+(1-r-s+2 r s) H+(1-s) r R, \\
H^{2} & =\frac{1}{4}(1-r+s)^{2} D+\frac{1}{2}(1-r+s)(1+r-s) H+\frac{1}{4}(1+r-s)^{2} R .
\end{aligned}
$$

If $a, b$, and $c$ are chosen as a basis where $a=D, b=D-H$, and 
$c=D-2 H+R$ we obtain:

$$
\begin{array}{ll}
a^{2}=a-2 r b+r^{2} c, & a b=\frac{1}{2}(1-r-s)(b-r c), \\
b^{2}=\frac{1}{4}(1-r-s)^{2} c, & a c=b c=c^{2}=0 .
\end{array}
$$

Thus this is also a special train algebra.

\section{Sex Linkage}

We shall use the subscripts 1 and 2 for the haploid (usually male) and diploid sex respectively. The basis elements, representing genotypes, will thus be called $D_{1}, R_{1}, D_{2}, H_{2}, R_{2}$. Since members of the same sex do not produce offspring it is convenient to define the product of any two basis elements with the same subscript as zero. The rest of the table is:

$$
\begin{aligned}
& D_{1} D_{2}=\frac{1}{2}\left(D_{1}+D_{2}\right), D_{1} R_{2}=\frac{1}{2}\left(R_{1}+H_{2}\right), D_{1} H_{2}=\frac{1}{4}\left(D_{1}+R_{1}+D_{2}+H_{2}\right), \\
& R_{1} R_{2}=\frac{1}{2}\left(R_{1}+R_{2}\right), R_{1} D_{2}=\frac{1}{2}\left(D_{1}+H_{2}\right), R_{1} H_{2}=\frac{1}{4}\left(D_{1}+R_{1}+H_{2}+R_{2}\right) .
\end{aligned}
$$

The algebra is certainly not a special train algebra. In fact, the existence of a basis consisting entirely of nilpotent elements makes it clear that no non-trivial homomorphism into the reals exists (i.e. the algebra is not baric in the terminology of (1)).

A simplification can be effected by using as a basis $D_{1}, a=D_{1}+D_{2}$, $b=D_{1}-R_{1}, c=D_{2}-R_{2}, d=D_{2}-2 H_{2}+R_{2}$. The table then becomes

$$
\begin{aligned}
& D_{1}^{2}=0, a D_{1}=\frac{1}{2} a, b D_{1}=0, c D_{1}=\frac{1}{2} b+\frac{1}{4} c+\frac{1}{4} d, d D_{1}=0, \\
& a^{2}=a, a b=\frac{1}{4} c+\frac{1}{4} d, a c=\frac{1}{2} b+\frac{1}{4} c+\frac{1}{4} d, a d=0, b^{2}=0, b c=\frac{1}{2} d, \\
& b d=0, c^{2}=0, c d=0, d^{2}=0 .
\end{aligned}
$$

It follows immediately from the table that the subspace $\{a, b, c, d\}$ is an ideal containing the product of any two elements in the original ring. This ideal is the set of all elements for which the sums of the coefficients of the original basis elements of subscripts one and two are equal. (The genetic significance of the ideal is that it contains all the elements which represent populations.) Furthermore, the ideal is a special train algebra. This can be more easily seen if $b+c$ is used as a basis element instead of $c$. It is interesting to note that the powers of the ideal $\{b, c, d\},\left(\{b, c, d\}^{2}=d,\{b, c, d\}^{3}=0\right.$ ) are ideals even in the original ring.

The ideas in $\S 2$ may be used to show that the elements of the form $a+y b+y c+\left(y^{2}+y\right) d$ represent all the non-zero idempotents and that the plenary powers of an element of weight one approach an idempotent. The elements $A$ which are non-zero and satisfy $\left(A^{2}\right)^{2}=A^{2}$ are precisely those of the form $a+y b+y c+x d$ where $x$ and $y$ are arbitrary.

The genetic significance of these results can be made clear by expressing them in terms of the original basis. By letting $y=-\beta$ and $\alpha=1-\beta$ we find that the idempotents are exactly all those elements $\alpha D_{1}+\beta R_{1}+\alpha^{2} D_{2}+$ $2 \alpha \beta H_{2}+\beta^{2} R_{2}$ with $\alpha+\beta=1$. 


\section{SPECIAL TRAIN ALGEBRAS ARISING IN GENETICS}

It clearly follows that the elements which satisfy $\left(A^{2}\right)^{2}=A^{2}$ are precisely those of the form

$$
\alpha D_{1}+\beta R_{1}+\left(\alpha^{2}+\gamma\right) D_{2}+(2 \alpha \beta-2 \gamma) H_{2}+\left(\beta^{2}+\gamma\right) R_{2} \text { where } \gamma=x-\left(y^{2}+y\right) .
$$

A more intuitive feeling for this result can be grasped if it is noted that $\gamma\left(\mathrm{D}_{2}-2 \mathrm{H}_{2}+\mathrm{R}_{2}\right)$ is the most general element which can be added to a genotype distribution which will not change the distribution of gametes produced. For a more classical approach see (9).

\section{Sex Linkage with Mutations}

Using the same style as before, the table is:

$$
\begin{aligned}
D_{1} D_{2}= & \left.\frac{1}{2}\left[(1-r) D_{1}+r R_{1}+(1-r)^{2} D_{2}+2 r(1-r) H_{2}+r^{2} R_{2}\right)\right], \\
R_{1} R_{2}= & \frac{1}{2}\left[s D_{1}+(1-s) R_{1}+s^{2} D_{2}+2 s(1-s) H_{2}+(1-s)^{2} R_{2}\right], \\
R_{1} D_{2}= & \frac{1}{2}\left[(1-r) D_{1}+r R_{1}+s(1-r) D_{2}+\{r s+(1-r)(1-s)\} H_{2}+r(1-s) R_{2}\right], \\
D_{1} R_{2}= & \frac{1}{2}\left[s D_{1}+(1-s) R_{1}+s(1-r) D_{2}+\{r s+(1-r)(1-s)\} H_{2}+r(1-s) R_{2}\right], \\
D_{1} H_{2}= & \frac{1}{4}\left[(1-r+s) D_{1}+(1+r-s) R_{1}+(1-r)(1-r+s) D_{2}+\{1+(1-2 r)(r-s)\} H_{2}\right. \\
& \left.\quad+r(1+r-s) R_{2}\right], \\
R_{1} H_{2}= & \frac{1}{4}\left[(1-r+s) D_{1}+(1+r-s) R_{1}+s(1-r+s) D_{2}+\{1+(1-2 s)(s-r)\} H_{2}\right. \\
& \left.\quad+(1-s)(1+r-s) R_{2}\right] .
\end{aligned}
$$

Again the table is simplified by using $D_{1}, a, b, c, d$ as a basis.

$$
\begin{aligned}
D_{1}^{2} & =0, a D_{1}=\frac{1}{2} a-\frac{1}{2} r b-\frac{1}{2} r c-\frac{1}{2} r(1-r) d, b D_{1}=0, \\
c D_{1} & =\frac{1}{2}(1-r-s) b+\frac{1}{4}(1-r-s) c+\frac{1}{4}(1-2 r)(1-r-s) d, d D_{1}=0, \\
a^{2} & =a-r b-r c-r(1-r) d, a b=\frac{1}{4}(1-r-s) c+\frac{1}{4}(1-2 r)(1-r-s) d, \\
a c & =\frac{1}{2}(1-r-s) b+\frac{1}{4}(1-r-s) c+\frac{1}{4}(1-2 r)(1-r-s) d, a d=0 \\
b^{2} & =0, b c=\frac{1}{2}(1-r-s)^{2} d, b d=0, c^{2}=0, c d=0, d^{2}=0 .
\end{aligned}
$$

Again $\{a, b, c, d\}$ is an ideal containing the product of any two elements in the ring. Furthermore, the ideal is a special train algebra, containing a unique non-zero idempotent unless $r+s=0$. As in the case of no sex linkage, the genotype distributions approach a stable distribution unless $r=s=1$.

\section{Polyploidy}

We shall first consider the gametic $n$-ploidy algebra. The types will be denoted by $D_{n}, D_{n-1}, \ldots, D_{0}$ where $D_{i}$ stands for the type which has $i$ dominant genes and $n-i$ recessive genes. The multiplication table is:

$$
D_{a} D_{b}=\left(\begin{array}{c}
2 n \\
n
\end{array}\right)^{-1} \sum_{i=0}^{n}\left(\begin{array}{c}
a+b \\
i
\end{array}\right)\left(\begin{array}{c}
2 n-a-b \\
n-i
\end{array}\right) D_{i}
$$

where $\left(\begin{array}{l}n \\ r\end{array}\right)$ stands for the usual binomial coefficient. (It is convenient to use 
the symbol $\left(\begin{array}{l}n \\ r\end{array}\right)$ even when $r<0$ or $r>n$ in which case we define $\left(\begin{array}{l}n \\ r\end{array}\right)=0$. It is then easily seen that the identity $\left(\begin{array}{l}n \\ r\end{array}\right)+\left(\begin{array}{c}n \\ r-1\end{array}\right)=\left(\begin{array}{c}n+1 \\ r\end{array}\right)$ is valid in general.)

Lemma 6.1.

$$
\begin{aligned}
\sum_{r=0}^{s}(-1)^{r}\left(\begin{array}{c}
s \\
r
\end{array}\right) P_{2 n-r} & =\left(\begin{array}{c}
n \\
s
\end{array}\right)\left(\begin{array}{c}
2 n \\
s
\end{array}\right)^{-1}\left[\sum_{r=0}^{s}(-1)^{r}\left(\begin{array}{l}
s \\
r
\end{array}\right) D_{n-r}\right] \text { for } 0 \leqq s \leqq n \\
& =0 \text { for } n<s \leqq 2 n,
\end{aligned}
$$

where $P_{t}$ is defined as $\left(\begin{array}{c}2 n \\ n\end{array}\right)^{-1} \sum_{i=0}^{n}\left(\begin{array}{l}t \\ i\end{array}\right)\left(\begin{array}{c}2 n-t \\ n-i\end{array}\right) D_{i}$ for $0 \leqq t \leqq 2 n$.

$\left(P_{t}=D_{a} D_{b}\right.$ for any $a, b$ satisfying $a+b=t$.)

Proof. By definition

$$
\sum_{r=0}^{s}(-1)^{r}\left(\begin{array}{l}
s \\
r
\end{array}\right) P_{2 n-r}=\left(\begin{array}{c}
2 n \\
n
\end{array}\right)^{-1} \sum_{r=0}^{s} \sum_{i=0}^{n}(-1)^{r}\left(\begin{array}{l}
s \\
r
\end{array}\right)\left(\begin{array}{c}
2 n-r \\
i
\end{array}\right)\left(\begin{array}{c}
r \\
n-i
\end{array}\right) D_{i} .
$$

Using the fact that

$$
\left(\begin{array}{l}
s \\
r
\end{array}\right)\left(\begin{array}{l}
r \\
p
\end{array}\right)=\left(\begin{array}{l}
s \\
p
\end{array}\right)\left(\begin{array}{l}
s-p \\
r-p
\end{array}\right)
$$

the latter expression becomes

$$
\left(\begin{array}{c}
2 n \\
n
\end{array}\right)^{-1} \sum_{r=0}^{s} \sum_{i=0}^{n}(-1)^{r}\left(\begin{array}{c}
2 n-r \\
i
\end{array}\right)\left(\begin{array}{c}
s \\
n-i
\end{array}\right)\left(\begin{array}{c}
s-(n-i) \\
r-(n-i)
\end{array}\right) D_{i} .
$$

From the identity $\left(\begin{array}{c}n \\ i\end{array}\right)-\left(\begin{array}{c}n-1 \\ i\end{array}\right)=\left(\begin{array}{c}n-1 \\ i-1\end{array}\right)$ it may be obtained by induction on $s$ that $\sum_{t=0}^{s}(-1)^{t}\left(\begin{array}{c}s \\ t\end{array}\right)\left(\begin{array}{c}n-t \\ i\end{array}\right)=\left(\begin{array}{c}n-s \\ i-s\end{array}\right)$. Replacing $n$ by $n+1$ and $s$ by $s-(n-i)$ this becomes $\sum_{t=0}^{s-(n-i)}(-1)^{t}\left(\begin{array}{c}s-(n-i) \\ t\end{array}\right)\left(\begin{array}{c}n+i-t \\ i\end{array}\right)=\left(\begin{array}{c}2 n-s \\ n-s\end{array}\right)$. We now let $t=r-(n-i)$ and sum over $r$. The relationship becomes:

$$
\sum_{r=n-i}^{s}(-1)^{r-(n-i)}\left(\begin{array}{c}
s-(n-i) \\
r-(n-i)
\end{array}\right)\left(\begin{array}{c}
2 n-r \\
i
\end{array}\right)=\left(\begin{array}{c}
2 n-s \\
n-s
\end{array}\right) \text {. }
$$

Since $\left(\begin{array}{l}s-(n-i) \\ r-(n-i)\end{array}\right)=0$ for $r<n-i$ in view of our convention, the summation may be considered as going from 0 to $s$. Expression (1) now simplifies to $\left(\begin{array}{c}2 n \\ n\end{array}\right)^{-1} \sum_{i=0}^{n}(-1)^{n-i}\left(\begin{array}{c}s \\ n-i\end{array}\right)\left(\begin{array}{c}2 n-s \\ n-s\end{array}\right) D_{i}=\left(\begin{array}{c}2 n \\ n\end{array}\right)^{-1} \sum_{i=0}^{n}(-1)^{i}\left(\begin{array}{c}s \\ i\end{array}\right)\left(\begin{array}{c}2 n-s \\ n-s\end{array}\right) D_{n-i}$. 
Since $\left(\begin{array}{c}2 n \\ n\end{array}\right)\left(\begin{array}{c}n \\ s\end{array}\right)=\left(\begin{array}{c}2 n \\ s\end{array}\right)\left(\begin{array}{c}2 n-s \\ n-s\end{array}\right)$ this becomes

$$
\left(\begin{array}{c}
2 n \\
s
\end{array}\right)^{-1}\left(\begin{array}{c}
n \\
s
\end{array}\right) \sum_{i=0}^{n}(-1)^{i}\left(\begin{array}{l}
s \\
i
\end{array}\right) D_{n-i}
$$

(Note the convenience of using the symbol $\left(\begin{array}{l}n \\ r\end{array}\right)$ for all integers $n$ and $r$. Thus the proof is valid even when $s>n$ in which case the last line reduces to 0 .)

Lemma 6.2. If $c_{s}$ is defined as $\sum_{i=0}^{s}(-1)^{i}\left(\begin{array}{l}s \\ i\end{array}\right) D_{n-i}$ for $0 \leqq s \leqq n$, then

$$
\begin{aligned}
c_{i} c_{j} & =\left(\begin{array}{c}
2 n \\
i+j
\end{array}\right)^{-1}\left(\begin{array}{c}
n \\
i+j
\end{array}\right) c_{i+j} \text { for } i+j \leqq n \\
& =0 \text { for } i+j>n .
\end{aligned}
$$

Proof. By comparison with the binomial theorem it is easily seen that

$$
\left[\sum_{i=0}^{s}(-1)^{i}\left(\begin{array}{l}
s \\
i
\end{array}\right) D_{n-i}\right]\left[\sum_{i=0}^{t}(-1)^{i}\left(\begin{array}{l}
t \\
i
\end{array}\right) D_{n-i}\right]=\sum_{i=0}^{s+t}(-1)^{i}\left(\begin{array}{c}
s+t \\
i
\end{array}\right) P_{2 n-i}
$$

where $P_{t}$ is defined as in Lemma 6.1. The result now follows from Lemma 6.1.

As an immediate consequence of Lemma 6.2 we obtain the following:

Theorem 6.1. The gametic n-ploidy algebra is a special train algebra with train roots $\left(\begin{array}{c}2 n \\ i\end{array}\right)^{-1}\left(\begin{array}{c}n \\ i\end{array}\right)$ for $0 \leqq i \leqq n$. Since $\lambda_{0}=1, \lambda_{1}=\frac{1}{2}$ and all other $\lambda$ 's are less than $\frac{1}{2}$, the remarks in $\S 2$ may be applied to this case.

When $n=2$ this reduces to the well-known case of the tetraploidy gametic algebra with table

$$
c_{0}^{2}=c_{0}, c_{0} c_{1}=\frac{1}{2} c_{1}, c_{0} c_{2}=c_{1}^{2}=\frac{1}{6} c_{2}, c_{1} c_{2}=c_{2}^{2}=0(1) .
$$

By applying the technique of duplication (5) we can now consider the zygotic algebra. We shall identify $c_{i} c_{j}$ with fixed $i+j$. This corresponds to identifying all $D_{a} D_{b}$ with fixed $a+b$ which in turn corresponds genetically to disregarding the nature of the contributions of each gamete to the zygote. It will be convenient to use the symbol $d_{i}$ to stand for any product $c_{a} c_{b}$ with $a+b=i . d_{0}, d_{1}, \ldots d_{2 n}$ is then a basis of the algebra.

Theorem 6.2. The zygotic n-ploidy algebra is a special train algebra with basis elements $d_{i}, 0 \leqq i \leqq 2 n$ and multiplication table:

$$
\begin{aligned}
& d_{i} d_{j}=\left(\begin{array}{c}
2 n \\
i
\end{array}\right)^{-1}\left(\begin{array}{c}
2 n \\
j
\end{array}\right)^{-1}\left(\begin{array}{l}
n \\
i
\end{array}\right)\left(\begin{array}{l}
n \\
j
\end{array}\right) d_{i+j} \text { for } i, j \leqq n, \\
& d_{i} d_{j}=0 \text { otherwise. }
\end{aligned}
$$

Proof. The table follows immediately from the process of duplication 
and implies the special train property. The remarks in $\S 2$ remain valid in the zygotic case.

When $n=2$ the table becomes $d_{0}^{2}=d_{0}, d_{0} d_{1}=\frac{1}{2} d_{1}, d_{1}^{2}=\frac{1}{4} d_{2}, d_{0} d_{2}=\frac{1}{6} d_{2}$, $d_{1} d_{2}=\frac{1}{12} d_{3}, d_{2}^{2}=\frac{1}{36} d_{4}$ with all other products zero.

Lemma 6.3.

$$
\sum_{i=0}^{n} x_{i} D_{n-i}=\sum_{r=0}^{n}(-1)^{r}\left[\sum_{s=r}^{n}\left(\begin{array}{l}
s \\
r
\end{array}\right) x_{s}\right] c_{r}
$$

Proof. By using the definition $c_{r}=\sum_{i=0}^{r}(-1)^{i}\left(\begin{array}{c}r \\ i\end{array}\right) D_{n-i}$ we obtain for the right-hand side

$$
\sum_{r=0}^{n} \sum_{s=r}^{n} \sum_{i=0}^{r}(-1)^{i}(-1)^{r}\left(\begin{array}{l}
s \\
r
\end{array}\right)\left(\begin{array}{l}
r \\
i
\end{array}\right) x_{s} D_{n-i}
$$

The coefficient of $x_{s} D_{n-i}$ is

$$
(-1)^{i} \sum_{r=i}^{s}(-1)^{r}\left(\begin{array}{l}
s \\
r
\end{array}\right)\left(\begin{array}{l}
r \\
i
\end{array}\right)
$$

Now

$$
\sum_{r=i}^{s}(-1)^{r}\left(\begin{array}{l}
s \\
r
\end{array}\right)\left(\begin{array}{l}
r \\
i
\end{array}\right)=\sum_{r=i}^{s}(-1)^{r}\left(\begin{array}{l}
s \\
i
\end{array}\right)\left(\begin{array}{l}
s-i \\
r-i
\end{array}\right)=\left(\begin{array}{l}
s \\
i
\end{array}\right) \sum_{r=i}^{s}(-1)^{r}\left(\begin{array}{l}
s-i \\
r-i
\end{array}\right)=(-1)^{i} \delta_{i s}
$$

since the expression within the summation sign is simply an expansion of $(-1)^{i}(1-1)^{s-i}$. Thus the coefficient is $\delta_{i s \cdot}$ Hence the coefficient of $D_{n-i}$ is $x_{i}$. This completes the proof.

This lemma gives an explicit formula for change of basis. In the special case where $x_{i}=1$ and all other $x$ 's are 0 the lemma becomes

$$
D_{n-i}=\sum_{r=0}^{i}(-1)^{r}\left(\begin{array}{l}
i \\
r
\end{array}\right) c_{r}
$$

In other words the relationship expressing the $D$ 's in terms of the $c$ 's is the same as the relationship expressing the $c$ 's in terms of the $D$ 's. For example in the simplest case where $c_{0}=D_{2}, c_{1}=D_{2}-D_{1}$ and $c_{2}=D_{2}-2 D_{1}+D_{0}$ we have $D_{2}=c_{0}, D_{1}=c_{0}-c_{1}$, and $D_{0}=c_{0}-2 c_{1}+c_{2}$.

The special case where $x_{i} \geqq 0$ for all $i$ and $\sum_{i=0}^{n} x_{i}=1$ corresponds to a distribution of gametic types. $\left(\sum_{i=0}^{n} x_{i} D_{n-i}\right.$ corresponds to the distribution in which $x_{i}$ is the fraction of types which have exactly $i$ recessive genes.) If a package of $i$ genes is chosen at random from one individual of the population, the probability of all genes being recessive is

$$
\left(\begin{array}{l}
n \\
i
\end{array}\right)^{-1} \sum_{s=i}^{n}\left(\begin{array}{l}
s \\
i
\end{array}\right) x_{s}
$$


If the probability is denoted by $y_{i}$, then it follows from Lemma 6.3 that

$$
\sum_{i=0}^{n} x_{i} D_{n-i}=\sum_{i=0}^{n}(-1)^{i}\left(\begin{array}{c}
n \\
i
\end{array}\right) y_{i} c_{i} \text {. }
$$

Theorem 6.3. A necessary and sufficient condition for a non-zero element to be idempotent is that $y_{i}=y_{1}^{i}$ for all $i$ satisfying $0 \leqq i \leqq n$. Otherwise no plenary power of the element is idempotent.

Proof. We first prove the sufficiency of the first statement.

$$
\text { Since }\left[\sum_{i=0}^{n}(-1)^{i}\left(\begin{array}{c}
n \\
i
\end{array}\right) y_{i} c_{i}\right]^{2}=\sum_{i=0}^{n} \sum_{j=0}^{n}(-1)^{i+j}\left(\begin{array}{c}
n \\
i
\end{array}\right)\left(\begin{array}{l}
n \\
j
\end{array}\right) y_{i} y_{j} c_{i} c_{j}
$$

and

$$
c_{i} c_{j}=\left(\begin{array}{c}
2 n \\
i+j
\end{array}\right)^{-1}\left(\begin{array}{c}
n \\
i+j
\end{array}\right) c_{i+j}
$$

the coefficient of $c_{k}$ in the product becomes

$$
\sum_{i=0}^{k}(-1)^{k}\left(\begin{array}{c}
n \\
i
\end{array}\right)\left(\begin{array}{c}
n \\
k-i
\end{array}\right) y_{i} y_{k-i}\left(\begin{array}{c}
2 n \\
k
\end{array}\right)^{-1}\left(\begin{array}{l}
n \\
k
\end{array}\right) \text {. }
$$

The use of the fact that

$$
\sum_{i=0}^{k}\left(\begin{array}{c}
n \\
i
\end{array}\right)\left(\begin{array}{c}
n \\
k-i
\end{array}\right)=\left(\begin{array}{c}
2 n \\
k
\end{array}\right)
$$

completes the proof. (The latter is verified by comparing the coefficient of $x^{k}$ in $(1+x)^{2 n}$ and in $(1+x)^{n}(1+x)^{n}$.) The necessity follows from the fact that the value of $y_{1}$ determines the idempotent uniquely.

The last part follows from a careful examination of the proof of Theorem 2.2. The basic idea is that if, in a recursion relation of the form $A_{n+1}=\lambda A_{n}+B$ for $\lambda \neq 0, A_{1} \neq A_{2}$, then $A_{n} \neq A_{n+1}$ for all $n$.

If the idempotent $\sum_{i=0}^{n}(-1)^{i}\left(\begin{array}{c}n \\ i\end{array}\right) y_{1}^{i} c_{i}$ is expressed in terms of $D$ 's, the coefficient of $D_{n-j}$ becomes $\left(\begin{array}{l}n \\ j\end{array}\right) y_{1}^{j}\left(1-y_{1}\right)^{n-j}$. Thus the corresponding genotype distribution is a binomial distribution as seems intuitively reasonable.

Analogous results apply to the zygotic algebra. Let us use the symbol $D_{i}$ to stand for any product $D_{a} D_{b}$ with $a+b=i$. No confusion should arise if the zygotic and gametic algebras are not discussed simultaneously. $D_{2 n}$, $D_{2 n-1}, \ldots, D_{0}$ is a basis of the algebra where $D_{i}$ stands for the type which has $i$ dominant genes and $2 n-i$ recessive genes. It is easily seen that the $D$ 's and the $d$ 's bear the same relation to each other as the $D$ 's and the $c$ 's of the gametic algebra provided that $n$ is replaced by $2 n$. Thus we have

$$
d_{s}=\sum_{i=0}^{s}(-1)^{i}\left(\begin{array}{l}
s \\
i
\end{array}\right) D_{2 n-i}
$$

E.M.S. -D 
as well as the analogue of Lemma 6.3. Furthermore we have:

Theorem 6.4. A necessary and sufficient condition for a non-zero element to be an idempotent is that $y_{i}=y_{1}^{i}$ for all $i$ satisfying $0 \leqq i \leqq 2 n$. If $y_{i}=y_{1}^{i}$ for all $i$ satisfying $0 \leqq i \leqq n$ then the element satisfies $\left(A^{2}\right)^{2}=A^{2}$. Otherwise no plenary power of the element is idempotent.

Proof. As in the proof of Theorem 6.3 we consider

$$
\left[\sum_{i=0}^{2 n}(-1)^{i}\left(\begin{array}{c}
2 n \\
i
\end{array}\right) y_{i} d_{i}\right]^{2}=\sum_{i=0}^{2 n} \sum_{j=0}^{2 n}(-1)^{i+j}\left(\begin{array}{c}
2 n \\
i
\end{array}\right)\left(\begin{array}{c}
2 n \\
j
\end{array}\right) y_{i} y_{j} d_{i} d_{j} .
$$

Using the formula

$$
d_{i} d_{j}=\left(\begin{array}{c}
2 n \\
i
\end{array}\right)^{-1}\left(\begin{array}{c}
2 n \\
j
\end{array}\right)^{-1}\left(\begin{array}{l}
n \\
i
\end{array}\right)\left(\begin{array}{l}
n \\
j
\end{array}\right) d_{i+j},
$$

the coefficient of $d_{k}$ in the product becomes

$$
\begin{aligned}
\sum_{i=0}^{k}(-1)^{k}\left(\begin{array}{c}
2 n \\
i
\end{array}\right)\left(\begin{array}{c}
2 n \\
k-i
\end{array}\right) y_{i} y_{k-i}\left(\begin{array}{c}
2 n \\
i
\end{array}\right)^{-1}\left(\begin{array}{c}
2 n \\
k-i
\end{array}\right)^{-1}\left(\begin{array}{c}
n \\
i
\end{array}\right)\left(\begin{array}{c}
n \\
k-i
\end{array}\right) & =\sum_{i=0}^{k}(-1)^{k}\left(\begin{array}{c}
n \\
i
\end{array}\right)\left(\begin{array}{c}
n \\
k-i
\end{array}\right) y_{1}^{k} .
\end{aligned}
$$

The rest of the proof is similar to the gametic case except for the consideration of the property $\left(A^{2}\right)^{2}=A^{2}$.

Since the coefficient of $d_{i}$ in a product does not depend on the coefficient of $d_{j}$ for $j>i$, the condition $y_{i}=y_{1}^{i}$ for $0 \leqq i \leqq n$ will at least guarantee that the coefficient of $d_{i}$ for $i \leqq n$ will be the same for $A$ and $A^{2}$. Since $d_{i} d_{j}=0$ for $i>n$ it follows that $A^{2}$ depends only on the coefficients of $d_{i}$ for $i \leqq n$ in $A$. Hence $A^{2}=\left(A^{2}\right)^{2}$.

As before it may be noted that

$$
\sum_{i=0}^{2 n}(-1)^{i}\left(\begin{array}{c}
2 n \\
i
\end{array}\right) y_{1}^{i} d_{i}=\sum_{j=0}^{2 n}\left(\begin{array}{c}
2 n \\
j
\end{array}\right) y_{1}^{j}\left(1-y_{1}\right)^{2 n-j} D_{2 n-j}
$$

For a more classical discussion of polyploidy see (7) and (8).

\section{Polyploidy with Mutations}

Instead of discussing the product directly it will be convenient to regard the product as being obtained from the product with no mutations by a linear mapping which we call the mutation mapping. To be more explicit $D_{a} D_{b}=T\left(D_{a} \times D_{b}\right)$ where $D_{a} \times D_{b}$ is the product as defined in $\S 6$ and $T$ is the linear mapping satisfying

$$
T\left(D_{i}\right)=\left[\sum_{u+v=t}\left(\begin{array}{c}
i \\
u
\end{array}\right)\left(\begin{array}{c}
n-i \\
v
\end{array}\right)(1-r)^{u} r^{i-u}(1-s)^{n-i-v} s^{v}\right] D_{t} .
$$

The mapping is obtained by elementary reasoning in probability. Since the method applies equally well to the gametic and zygotic algebra we simply use $n$. 
(Our previous notation would require $2 n$ for the zygotic case.) Note that the coefficient of $D_{t}$ is the coefficient of $x^{t}$ in the expansion of

$$
[(1-r) x+r]^{i}[s x+(1-s)]^{n-i} \text {. }
$$

This formulation will be useful in the sequel. Let

$$
[(1-r) x+r]^{i}[s x+(1-s)]^{n-i}=\sum_{t=0}^{n} a_{i t} x^{t} .
$$

Writing $T\left(D_{i}\right)=\sum_{u=0}^{n} a_{i u} D_{u}$ it follows from Lemma 6.3 that

$$
T\left(D_{i}\right)=\sum_{v=0}^{n}(-1)^{v}\left[\sum_{u=0}^{n-v}\left(\begin{array}{c}
n-v \\
v
\end{array}\right) a_{i u} c_{v}\right]
$$

Now consider the expansion $[(1-r) x+r(x-1)]^{i}[s x+(1-s)(x-1)]^{n-i}$. This is $\sum_{u=0}^{n} a_{i u} x^{u}(x-1)^{n-u}$. The coefficient of $x^{n-v}$ is $(-1)^{i} \sum_{u=0}^{n-v}\left(\begin{array}{c}n-u \\ v\end{array}\right) a_{i u}$ which is the coefficient of $c_{v}$ in $T\left(D_{i}\right)$. Thus by simplifying the above expression we find that the coefficient of $c_{v}$ in $T\left(D_{i}\right)$ is the coefficient of $x^{n-v}$ in $(x-r)^{i}$ $[x-(1-s)]^{n-i}$. We call this coefficient $b_{i}$.

It follows that the coefficient of $c_{v}$ in $T\left(c_{w}\right)$ is $\sum_{i=0}^{w}(-1)^{i}\left(\begin{array}{c}w \\ i\end{array}\right) b_{n-i}$. Now consider $[x-r]^{n-w}[(x-r)-\{x-(1-s)\}]^{w}$. Expanding the second term by the binomial theorem we may write this as $\sum_{i=0}^{w}\left(\begin{array}{c}w \\ i\end{array}\right)(x-r)^{n-i}[x-(1-s)]^{i}$. Thus the coefficient of $x^{n-v}$ is $\sum_{i=0}^{w}(-1)^{i}\left(\begin{array}{c}w \\ i\end{array}\right) b_{n-i}$. This shows that the coefficient of $c_{v}$ in $T\left(c_{w}\right)$ is the coefficient of $x^{n-v}$ in $[x-r]^{n-w}[(x-r)-\{x-(1-s)\}]^{w}$ or $(x-r)^{n-w}(1-r-s)^{w}$. This finally proves the important result:

i.e.

$$
T\left(c_{w}\right)=(1-r-s)^{w} \sum_{v=w}^{n}(-1)^{v-w}\left(\begin{array}{c}
n-w \\
v-w
\end{array}\right) r^{v-w} c_{v}
$$

$$
T\left(c_{w}\right)=(1-r-s)^{w}\left[c_{w}-\left(\begin{array}{c}
n-w \\
1
\end{array}\right) r c_{w+1}+\left(\begin{array}{c}
n-w \\
2
\end{array}\right) r^{2} c_{w+2} \ldots\right] \text {. }
$$

Now that we have found $T$ explicitly in terms of the c's we can write down the multiplication table.

Theorem 7.1. The multiplication table of the n-ploidy gametic mutation algebra is

$$
\begin{aligned}
& c_{i} c_{j}=\left(\begin{array}{c}
2 n \\
i+j
\end{array}\right)^{-1}\left(\begin{array}{c}
n \\
i+j
\end{array}\right)(1-r-s)^{i+j}\left[c_{i+j}-\left(\begin{array}{c}
n-i-j \\
1
\end{array}\right) r c_{i+j+1}\right. \\
& \left.+\left(\begin{array}{c}
n-i-j \\
2
\end{array}\right) r^{2} c_{i+j+2}+\ldots\right] \text { for } i+j \leqq n,
\end{aligned}
$$

$=0$ otherwise.

E.M.S. $\rightarrow D 2$ 
A similar result is obtained for the zygotic algebra.

Theorem 7.2. The multiplication table of the n-ploidy zygotic mutation algebra is

$$
\begin{aligned}
& d_{i} d_{j}=\left(\begin{array}{c}
2 n \\
i
\end{array}\right)^{-1}\left(\begin{array}{c}
2 n \\
j
\end{array}\right)^{-1}\left(\begin{array}{l}
n \\
i
\end{array}\right)\left(\begin{array}{l}
n \\
j
\end{array}\right)(1-r-s)^{i+j} \\
& {\left[d_{i+j}-\left(\begin{array}{c}
n-i-j \\
1
\end{array}\right) r d_{i+j+1}+\left(\begin{array}{c}
n-i-j \\
2
\end{array}\right) r^{2} d_{i+j+2}-\ldots\right] \text { for } i, j \leqq n,}
\end{aligned}
$$

$=0$ otherwise.

The tables in $\S 3$ may be seen to be a special case of this. When $n=2$ the table in the zygotic case becomes

$$
\begin{aligned}
d_{0}^{2} & =d_{0}-4 r d_{1}+6 r^{2} d_{2}-4 r^{3} d_{3}+r^{4} d_{4}, \\
d_{0} d_{1} & =\frac{1}{2}(1-r-s)\left(d_{1}-3 r d_{2}+3 r^{2} d_{3}-r^{3} d_{4}\right), d_{1}^{2}=\frac{1}{4}(1-r-s)^{2}\left(d_{2}-2 r d_{3}+r^{2} d_{4}\right), \\
d_{0} d_{2} & =\frac{1}{6}(1-r-s)^{2}\left(d_{2}-2 r d_{3}+r^{2} d_{4}\right), d_{1} d_{2}=\frac{1}{12}(1-r-s)^{3}\left(d_{3}-r d_{4}\right), \\
d^{2} & =\frac{1}{36}(1-r-s)^{4} d_{4} \text { with all other products zero. }
\end{aligned}
$$

Corollary 1. The $n$-ploidy gametic and zygotic mutation algebras are special train algebras.

Since the train roots are $\left(\begin{array}{c}2 n \\ i\end{array}\right)^{-1}\left(\begin{array}{c}n \\ i\end{array}\right)(1-r-s)^{i}$, the results in $\S 2$ apply. A unique non-zero idempotent exists if $0<r+s \leqq 2$. Furthermore, the genotype distributions approach a stable distribution unless $r=s=1$.

The case $r+s=1$ is incredibly simple. In fact, if $e_{0}, c_{1}, c_{2}, \ldots, c_{n}$ is chosen as a basis where $e_{0}=c_{0}-\left(\begin{array}{l}n \\ 1\end{array}\right) r c_{1}+\left(\begin{array}{l}n \\ 2\end{array}\right) r^{2} c_{2}+\ldots+(-1)^{n} r^{n} c_{n}$, we obtain for the multiplication table that $e_{0}^{2}=e_{0}$ and that all other products of basis elements are zero! From this follows immediately

Corollary 2. If $r+s=1$, stability is obtained in one generation.

It is easily seen in this case that in terms of the $D$ 's the idempotent has the form $\sum_{i=0}^{n}\left(\begin{array}{l}n \\ i\end{array}\right) s^{i} r^{n-i} D_{i}$. Furthermore, these results are valid in the zygotic case as well.

In conclusion, the author wishes to thank the referee for many invaluable suggestions.

\section{REFERENCES}

(1) I. M. H. Etherington, Genetic algebras, Proc. Roy. Soc. Edin., 59 (1939), 242-258.

(2) I. M. H. Etherington, Commutative train algebras of ranks 2 and $3, J$. London Math. Soc., 15 (1940), 136-149; 20 (1945), 238.

(3) I. M. H. Etherington, Special train algebras, Quart. J. Math. (Oxford), 12 (1941), 1-8. 
(4) I. M. H. Etherington, Non-associative algebra and the symbolism of genetics, Proc. Roy. Soc. Edin. (B), 61 (1941), 24-42.

(5) I. M. H. Etherington, Duplication of linear algebras, Proc. Edin. Math. Soc. (2), 6 (1941), 222-230.

(6) I. M. H. ETHERINGTON, Non-commutative train algebras of ranks 2 and 3, Proc. London Math. Soc. (2), 52 (1951), 241-252.

(7) H. GeIRINGer, On some mathematical problems arising in the development of Mendelian genetics, J. Amer. Stat. Assoc., 44 (1949), 526-547.

(8) J. B. S. Haldane, Theoretical genetics of autopolyploids, J. Genetics, 22 (1930), 359-372.

(9) H. S. JENnings, The numerical results of diverse systems of breeding, Genetics, 1 (1916), 53-89.

(10) K. KNopp, Theory and Application of Infinite Series (Blackie), 1928.

(11) R. RAFFin, Axiomatisation des algèbres génétiques, Acad. Roy. Belgique Bull. Cl. Sci. (5), 37 (1951), 359-366.

(12) R. D. SchafER, Structure of genetic algebras, Amer. J. Math., 71 (1949), 121-135.

Mathematics Department

RUTGERS UNIVERSITY

NEW BRUNSWICK, N.J.

U.S.A. 